\title{
Use of Core Needle Biopsy rather than Fine-Needle Aspiration Cytology in the Diagnostic Approach of Breast Cancer
}

\author{
Paola Pagni Flaminia Spunticchia Simona Barberi Giuliana Caprio \\ Carlo Paglicci
}

DAI Ematologia, Oncologia, Anatomia Patologica e Medicina Territoriale, University of Rome 'La Sapienza', Rome, Italy

\section{Key Words}

Core needle biopsy · Fine-needle aspiration cytology · Breast cancer prevention

\begin{abstract}
Background and Aims: In the following study case, we reviewed breast ultrasound-guided core needle biopsy (CNB), using Mammotome (vacuum-assisted breast biopsy) and Tru-cut, carried out on palpable and nonpalpable uncertain breast lumps or malignant large lesions to be submitted to neoadjuvant chemotherapy. Material and Methods: Examinations were conducted during a 4-year period of clinical activity in a highly specialized center, from December 2009 to December 2013, in 712 patients previously subjected to fine-needle aspiration cytology (FNAC). Results: The results demonstrated that among the 712 breast biopsies, in many cases FNAC was not conclusive, and therefore we proceeded with the echo-guided biopsy, through which we were able to collect sufficient material for the histological examination in order to direct patients to surgery or follow-up. Conclusions: CNB is far superior to FNAC, especially in cases of uncertainty, where it is preferable to proceed directly with $C N B$, which may also determine additional prognostic and predictive markers. Initially FNAC is less expensive, but the actual costs involved tend to be higher for FNAC as it is less accurate and a CNB is often required. In accordance with recent publications, we can confirm the full validity of CNB in the diagnostic approach of breast lesions.
\end{abstract}




\section{Introduction}

Diagnosis of breast cancer is carried out by three medical members, the surgeon, who covers breast examination, the radiologist for mammography and ultrasound, and the pathologist for fine-needle aspiration cytology (FNAC) and core needle biopsy (CNB). Fineneedle aspiration biopsy (FNAB) has long been the most functional examination to determine the nature of the nodules, but gradually, CNB is being introduced [1-4]. The difference between the two techniques has been extensively studied, and several publications have highlighted the strengths and weaknesses of each $[5,6]$. CNB is used when FNAB is unable to give precise results, when there is a discordance between clinical, instrumental examination and cytology, or when it is preferable to obtain a histological examination of the lesion rather than a simple cytological examination (e.g. malignant lesions resulting from neoadjuvant chemotherapy).

\section{Advantages and Disadvantages of CNB}

CNB is certainly more reliable than cytology and less invasive than surgical biopsy, and allows you to plan therapeutic treatment. On the other hand, we have found it to be more expensive and invasive than cytology, and sometimes it is difficult to pinpoint subareolar injury or areas close to the pectoral muscle. There is also the risk of underestimating lesions in some cases $[7,8]$. This report covers the results of 4 years of experience with CNB at a center for the study, prevention and therapy of breast cancer.

Over the past few years CNB has been the first approach in cases of large lesions clearly malignant at mammography and/or ultrasound, where the future treatment will be neoadjuvant therapy. A CNB test was added in other cases after FNAB when the result was nondiagnostic or inconclusive. Whenever possible the procedure of FNAC and CNB was carried out a few days after the diagnosis of nodular lesion in order to minimize the patient's state of apprehension.

\section{Materials and Methods}

From 2009 to 2013, 712 breast biopsies were performed on women aged between 24 and 85 years (average age 55 years) visiting our center for screening or symptomatic consultancy. All patients above the age of 40 underwent clinical breast examination, mammography and ultrasound when requested by the radiologist; patients below the age of 40 were subjected to clinical breast examination and ultrasound only.

To ensure a correct classification and evaluation of the results, the diagnostic conclusions of the mammographies were classified into 5 categories, in accordance to both European and American recommendations (table 1). A similar classification was also used for the subsequent ultrasound examination (U) and for the definition of the classes 'cytological' (C) and 'histological' (B) in order to facilitate interdisciplinary communication.

All biopsies were performed under ultrasound guidance by experienced radiologists or sonographers dedicated to the prevention of breast cancer in an outpatient setting. Local anesthesia with the addition of adrenaline to reduce bleeding was used, and then a small incision was made to allow the insertion of the biopsy needle. In all cases, we attempted to withdraw 10 frustules, because, as claimed by some authors, increasing the number of biopsies increases the accuracy of the diagnosis, and in Brenner et al.'s case, the accuracy after 5 biopsies was $98 \%$ for masses [9-12]. This method was not always possible due to 
bleeding, and in the case of patients who were particularly apprehensive, we were unable to complete the procedure.

We used two techniques: vacuum-assisted breast biopsy (VABB) with 8-, 11- or 14gauge Mammotome probes (Devicor Medical Products, Inc.), or Tru-cut (Bard Magnum, Bard Biopsy Systems) with 12- or 14-gauge, 13-cm probes [13-16]. VABB was used whenever possible, but with very deep nodules adhering to the pectoral muscle or in the case of patients with very small breasts, Tru-cut was applied. VABB was found to be preferable as we were able to collect contiguous samples with a single introduction of the needle, and the quantity of tissue retrieved was greater. Therefore, the majority of pathologists prefer this technique, and it is much easier to locate the biopsied point.

In 165 cases, a nonmagnetic clip (MammoMARK, Devicor Medical) was placed to locate the lesion in the subsequent follow-up, when the lesion was too small and therefore difficult to find in the event of a subsequent surgery or in patients who are candidates for neoadjuvant therapy in order to more efficiently monitor the effectiveness of the therapy.

According to our experience, the average time to perform the biopsy was 20 min with a peak of $60 \mathrm{~min}$ from the beginning. Immediately after the procedure, synthetic ice was positioned upon the biopsy area of the patient for a few hours to prevent any bleeding. In the beginning, we tried to completely remove some injuries. Further on, this idea was abandoned as after the 6-month checkup, many patients were reported to have small residual nodules which had not been detected at the time of procedure.

\section{Results}

From December 1, 2009 to December 31, 2013, ultrasound-guided biopsies were performed in 712 women aged between 24 and 85 years (average age 55 years). The biopsied nodules were of variable dimensions ranging from 10 to $50 \mathrm{~mm}: 29.3 \%$ were less than $1 \mathrm{~cm}$ in size, $53.6 \%$ were between 10 and $19 \mathrm{~mm}, 14.4 \%$ between 20 and $39 \mathrm{~mm}$ and only $2.7 \%$ of the total tested were larger than $40 \mathrm{~mm}$.

VABB was used whenever possible and Tru-cut in the case of very deep nodules adhering to the pectoral muscle or in case of patients with very small breasts. A total of $556 \mathrm{VABB}$ probes were carried out (496 probes using a caliber of 11 gauge, 47 using 14-gauge probes, and 13 using 8-gauge probes) and 156 Tru-cut (94 probes of 12 gauge, 61 probes of 14 gauge, and 1 probe of 16 gauge, all with a length of $13 \mathrm{~cm}$ ).

The following number of frustules were sent for histological examination: 4-10 frustules in 238 cases, 11-20 frustules in 327 cases, 21-30 frustules in 142 cases and 31-40 frustules in 5 cases. In 141 patients, more than 10 frustules were taken in an attempt to completely remove the lesion using VABB with an 11- or 8-gauge probe, but complete removal occurred only in 93 cases, while in the remaining 48 cases, after the ultrasound-guided follow-up 6 months later, minimum residue of the lesion was still present [17-20].

In 165 cases, a nonmagnetic metal clip was placed (MammoMARK, Devicor Medical, characterized by a bioabsorbable collage capsule in integrated titanium wire marker in nonabsorbable radio-opaque compatible with an MRI field of 3.0 tesla or less), useful for the retrieval of examination specimens in the subsequent follow-up.

Breast biopsy under ultrasound guidance is a relatively quick method, being both safe and simple to perform. Complications in our center were minimal: in $91 \%$ of cases, no complications were found, in $8.7 \%$ of cases, hematoma was present, disappearing within a few days, and in only $0.3 \%$, lymphangitis was diagnosed. 
In figures 1, 2 and 3, all positive biopsies were compared with mammography, ultrasound and cytology to assess which method was more reliable in the diagnosis of a malignant disease. Figure 1 shows that in $63 \%$ of patients with a diagnosis of mammographic R5, the diagnosis was confirmed by biopsy, in $7 \%$ of patients, the breast was too dense to highlight the lesion (R1), and in 31\%, mammography detected uncertain lesions with benign prevalence (R3) to be later confirmed after histological sampling [21]. 35\% of the lumps that were non-diagnostic or benign at cytological examination (C1, C2) had a positive biopsy, $16 \%$ of C3 were neoplastic lesions, in 49\% of cases in which cytology identified the nodule as suspicious or a certainty for malignancy $(\mathrm{C} 4, \mathrm{C} 5)$, the diagnosis was confirmed by biopsy (fig. 2). Figure 3 shows all the cases with positive histology and the correlation with ultrasound. Among the $11 \%$ of the nodules classified as uncertain on ultrasound (U3), $10 \%$ were found to be malignant on histological examination. Only in $1 \%$ of the cases was the CNB unable to resolve the uncertainty, and the patients were sent to surgery; $89 \%$ of those suspected positive to ultrasound (U4-U5) were confirmed at histological examination.

\section{Discussion}

In the diagnostic path of benign and malignant lesions of the breast, there is currently increasing recourse to methods of biopsy of palpable or nonpalpable lesions in order to determine those who will definitely need surgery [22]. When cytological examination is insufficient to provide a diagnosis or is not adapt as in large lesions (generally more than 2 $\mathrm{cm}$ ) that will undergo neoadjuvant therapy, the chosen method is breast biopsy, generally ultrasound-guided.

Currently, there are essentially two methods used for breast biopsies: VABB and Trucut. In our series, we used VABB whenever possible and Tru-cut in the case of very deep nodules adhering to the pectoral muscle or in case of patients with very small breasts. The usage of VABB can extract contiguous samples with a single introduction of the needle, resulting in a lower risk of infection and less discomfort for the patient. In addition, more material is obtained due to the force of aspiration. In both cases, there is the possibility to leave a metal clip in position for the next 'search unit' lesion.

Over the past years, the use of breast biopsy has been a precise step in diagnostic and therapeutic breast lesions, as a method of second level after cytology failure, or as a method of first level in those lesions with ultrasound characteristics of sure malignancy or larger than $2 \mathrm{~cm}$ in size to be subjected to neoadjuvant chemotherapy [23].

Based on our experience on 712 consecutive breast biopsies performed over a range of 4 years, we can say that in many cases, FNAB was not conclusive, and therefore, we proceeded with the echo-guided biopsy, with which enough material was collected for histological examination, enabling us to direct patients to surgery or follow-up. The cost for FNAC was lower in the beginning, but the actual cost to obtain a definitive diagnosis was considerably higher for FNAC as it was less accurate and often backed up by further examinations such as CNB.

In accordance with recent publications, we conclude that, especially at a time when expenditure is constantly reviewed, CNB is far superior to FNAC in the diagnostic approach of breast cancer and, especially in cases of doubt, it is preferable to proceed directly with $\mathrm{CNB}$, which may also determine additional prognostic and predictive markers. 
Pagni et al.: Use of Core Needle Biopsy rather than Fine-Needle Aspiration Cytology in the Diagnostic Approach of Breast Cancer

\section{Disclosure Statement}

The authors declare that they have no conflict of interest in relation to this paper.

\section{References}

$\checkmark 1$ Westenend PJ, Sever AR, Beekman-De Volder HJ, Liem SJ: A comparison of aspiration cytology and core needle biopsy in the evaluation of breast lesions, Cancer 2001;93:146-150.

-2 Kline TS, Joshi LP, Neal HS: Fine-needle aspiration of the breast: diagnoses and pitfalls. A review of 3545 cases. Cancer 1979;44:1458-1464.

-3 Kooistra B, Wauters C, Strobbe L, Wobbes T: Preoperative cytological and histological diagnosis of breast lesions: a critical review. Eur J Surg Oncol 2010;36:934-940.

4 Parker SH, Jobe WE, Dennis MA, Stavros AT, Johnson KK, Yakes WF, Truell JE, Price JG, Kortz AB, Clark DG: US-guided automated large-core breast biopsy. Radiology 1993;187(2):507-511.

5 Willems SM, van Deurzen CH, van Diest PJ: Diagnosis of breast lesions: fine-needle aspiration cytology or core needle biopsy? A review. J Clin Pathol 2012;65:287-292.

6 Barra Ade A, Gobbi H, de L Rezende CA, Gouvea AP, de Lucena CE, Reis JH, Costa e Silva SZ: A comparison of aspiration cytology and core needle biopsy according to tumor size of suspicious breast lesions. Diagn Cytopathol 2008;36:26-31.

7 Brancato B, Crocetti E, Bianchi S, Catarsi S, Risso GG, Bulgaresi P, Piscioli F, Scialpi M, Ciatto S, Houssami N: Accuracy of needle biopsy of breast lesions visible on ultrasound: audit of fine needle versus core needle biopsy in 3233 consecutive samplings with ascertained outcome. Breast 2012;21:449-454.

8 Servizio Sanitario Regionale Emilia-Romagna: Protocollo diagnostico-terapeutico dello screening per la diagnosi precoce dei tumori della mammella: secondo livello diagnostico (assessment). Emilia Romagna, IV edizione, 2012, pp 16-18.

-9 Brenner RJ, Fajardo L, Fisher PR, Dershaw DD, Evans WP, Bassett L, Feig S, Mendelson E, Jackson V, Margolin FR: Percutaneous core biopsy of the breast: effect of operator experience and number of samples of diagnostic accuracy. Am J Roentgenol 1996;166(2):341-346.

10 Sauer G, Deissler H, Strunz K, Helms G, Remmel E, Koretz K, Terinde R, Kreienberg R: Ultrasound-guided large-core needle biopsies of breast lesions: analysis of 962 cases to determine the number of samples for reliable tumour classification. Br J Cancer 2005;92:231-235.

-11 Fishman JE, Milikowski C, Ramsinghani R, Velasquez MV, Avriam G: US-guided core-needle biopsy of the breast: how many specimens are necessary? Radiology 2003;226:779-782.

12 Liberman L, Dershaw DD, Rosen PP, Abramson AF, Deutch BM, Hann LE: Stereotactic 14-gauge breast biopsy: how many core biopsy specimens are needed? Radiology 1994;192:793-795.

13 Crystal P, Koretz M, Shcharynsky S, Makarov V, Strano S: Accuracy of sonographically guided 14-gauge coreneedle biopsy: results of 715 consecutive breast biopsies with at least two-year follow-up of benign lesions. J Clin Ultrasound 2005;33:47-52.

$\checkmark 14$ Povoski SP, Jimenez RE, Wang WP: Ultrasound-guided diagnostic breast biopsy methodology: retrospective comparison of the 8-gauge vacuum-assisted biopsy approach versus the spring-loaded 14-gauge core biopsy approach. World J Surg Oncol 2011;9:87.

15 Huber S, Wagner M, Medl M, Czembirek H: Benign breast lesions: minimally invasive vacuum-assisted biopsy with 11-gauge needles - patient acceptance and effect on follow-up imaging findings. Radiology 2003;226:783-790.

16 Buchberger W, Niehoff A, Obrist P, Rettl G, Dünser M: [Sonographically guided core needle biopsy of the breast: technique, accuracy and indications] Radiologe 2002;42:25-32. Article in German.

-17 Tennant SL, Evans A, Hamilton LJ, James J, Lee AH, Hodi Z, Ellis IO, Rakha EA, Wilson AR: Vacuum-assisted excision of breast lesions of uncertain malignant potential (B3) - an alternative to surgery in selected cases. Breast 2008;17:546-549.

18 Yom CK, Moon BI, Choe KJ, Choi HY, Park YL: Long-term results after excision of breast mass using a vacuum-assisted biopsy device. ANZ J Surg 2009;79:794-798.

19 Ko ES, Han H, Lee BH, Choe du H: Sonographic changes after removing all benign breast masses with sonographically guided vacuum-assisted biopsy. Acta Radiol 2009;50:968-974.

20 Wang ZL, Liu G, Li JL, Ding Q, Su L, Tang J, Ma L: Sonographically guided percutaneous excision of clinically benign breast masses. J Clin Ultrasound 2011;39:1-5.

21 Liberman L, Drotman M, Morris EA, LaTrenta LR, Abramson AF, Zakowski MF, Dershaw DD: Imaginghistologic discordance at percutaneous breast biopsy. Cancer 2000;89:2538-2546.

22 Ciatto S, Houssami N, Ambrogetti D, Bianchi S, Bonardi R, Brancato B, Catarzi S, Risso GG: Accuracy and underestimation of malignancy of breast core needle biopsy: the Florence experience of over 4000 consecutive biopsies. Breast Cancer Res Treat 2007;101:291-297. 
Pagni et al.: Use of Core Needle Biopsy rather than Fine-Needle Aspiration Cytology in the Diagnostic Approach of Breast Cancer

-23 Bolívar AV, Alonso-Bartolomé P, García EO, Ayensa FG: Ultrasound-guided core needle biopsy of nonpalpable breast lesions: a prospective analysis in 204 cases. Acta Radiol 2005;46:690-695.

Table 1. Mammography examination

R1 negative

R2 lesion with benign features

R3 the presence of abnormalities (doubtful, probably benign)

R4 abnormalities suspicious for malignancy (suspicious)

R5 malignant alterations (positive)

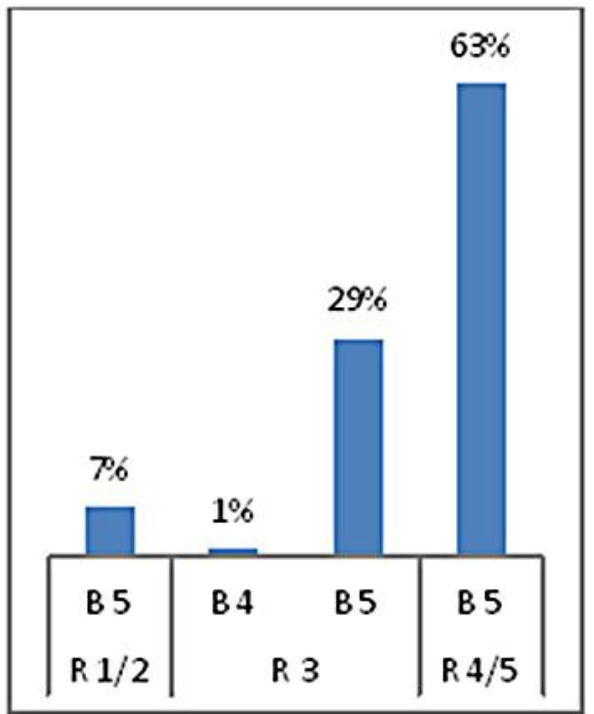

B: biopsy, R: mammography

Fig. 1. In $63 \%$ of patients with a diagnosis of mammographic R5, the diagnosis was confirmed by biopsy. In $7 \%$ of patients, the breast was too dense to highlight the lesion (R1). In 31\%, mammography detected uncertain lesions with a benign prevalence (R3) later to be confirmed with histological sampling. 
Pagni et al.: Use of Core Needle Biopsy rather than Fine-Needle Aspiration Cytology in the Diagnostic Approach of Breast Cancer

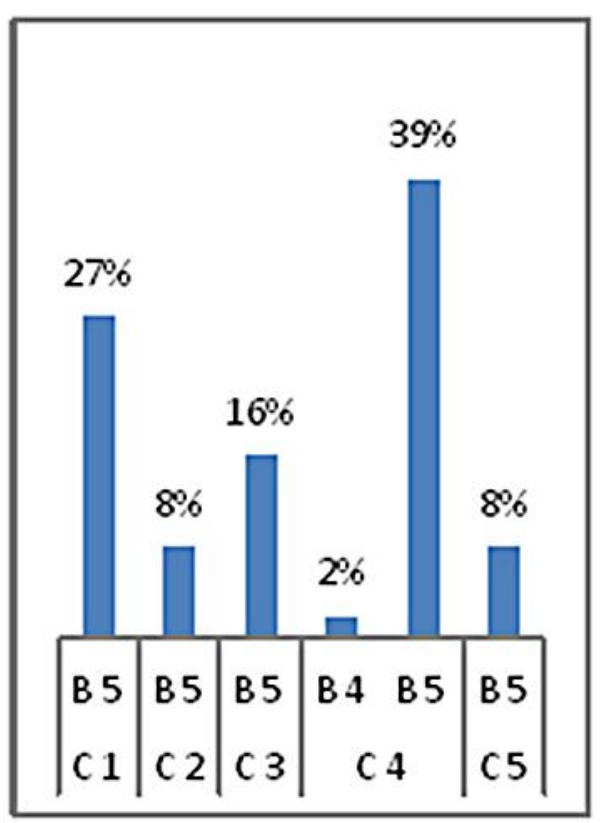

B: biopsy, C: citology

Fig. 2. 35\% of the lumps that were nondiagnostic or benign at cytological examination (C1-2) had a positive biopsy. $16 \%$ of C3 were neoplastic lesions. In $49 \%$ of cases in which cytology identified the nodule as suspicious or a certainty for malignancy (C4-5), the diagnosis was confirmed by biopsy.

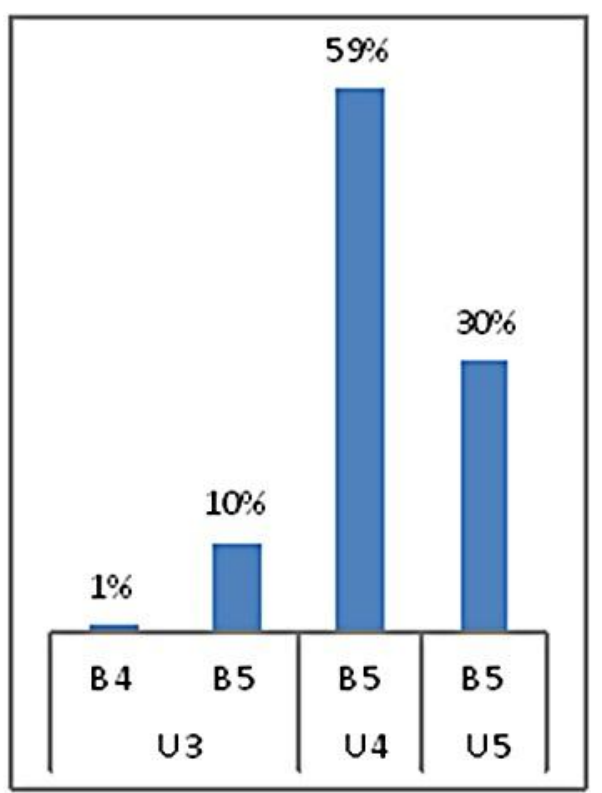

U: ultrasound, $B$ : biopsy

Fig. 3. All cases with positive histology and the correlation with ultrasound. Out of the $11 \%$ of the nodules classified as uncertain on ultrasound (U3), 10\% were found to be malignant on histological examination. Only in $1 \%$ of cases, the CNB was not able to resolve the uncertainty, and patients were sent to surgery. $89 \%$ of those suspected positive on ultrasound (U4-5) were confirmed at histological examination. 